\title{
Avalanche Photodiodes for the CMS Electromagnetic Calorimeter
}

\section{Q. Ingram*, K. Deiters, D. Renker, T. Sakhelashvili}

Paul Scherrer Institut, Villigen PSI, Switzerland

E-mail: 'quentin. ingram@psi.ch'

\section{A. Kuznetsov, Y. Musienko’ J. Swain, S Reucroft}

Northeastern University, Boston, USA

\section{Kronqvist, R. Rusack, A. Singovski, P.Vikas}

University of Minnesota, Minneapolis, USA

\section{Z. Antunovic, N. Godinovic, I. Soric}

University of Split, Split, Croatia

\begin{abstract}
The light from the lead tungstate crystals of the barrel part of the CMS ECAL will be measured using avalanche photodiodes developed for the purpose by Hamamatsu Photonics in collaboration with CMS. The requirements for the diodes are described, and the performance achieved is summarised, with emphasis on the long-term reliability in the difficult high radiation conditions at CMS.
\end{abstract}

\section{Introduction}

In the barrel part of the CMS ECAL, the light from each of 61,200 lead tungstate crystals will be detected by a pair of Avalanche Photodiodes (APDs) [i]1ㄱ. The use of lead tungstate allows a fast, compact and radiation hard calorimeter, but has the disadvantage that the amount of light produced by the crystals is modest. The calorimeter is located within a high field solenoid.

The requirements for the photodetectors include operation in a $4 \mathrm{~T}$ field, radiation hardness at the level of $2.10^{13} \mathrm{n} / \mathrm{cm}^{2}$ and $2.5 \mathrm{kGray}$, speed ( $\leq 10 \mathrm{nsec}$ ), and of course compatibility with the demanding ECAL energy resolution requirements. The latter implies large area, good sensitivity to the light emitted by the crystal (430 $\mathrm{nm}$ peak), stability and

${ }^{*}$ Speaker.

${ }^{\dagger}$ on leave from INR Moscow 

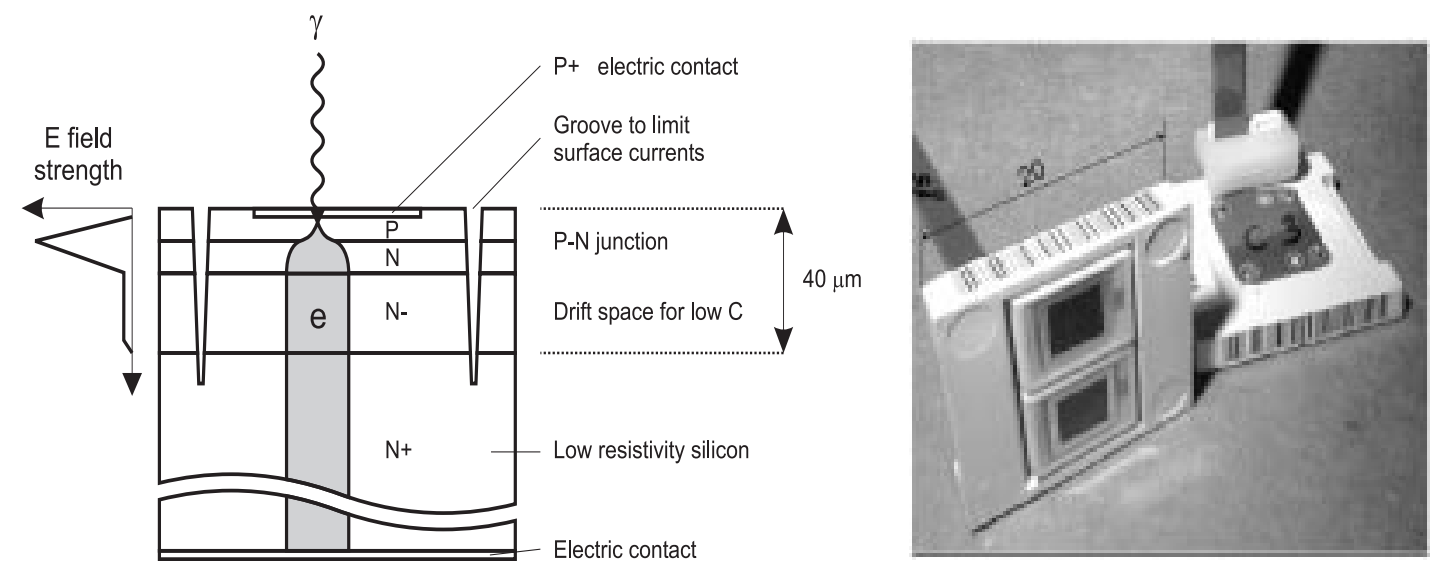

Figure 1: Schematic of the APD structure and two APDs ready for mounting on a crystal

insensitivity to voltage and temperature fluctuations as well as low capacitance, resistance, noise and dark current. However, the low light yield of the crystals brings in two further important requirements: gain and insensitivity to ionising particles traversing the diode. Finally they must be affordable since 122,400 pieces are needed.

\section{APD Characteristics}

The APDs to be used in CMS are silicon photodiodes operated in avalanche mode to provide gain. They have been developed over 8 years by Hamamatsu in close collaboration with CMS ECAL groups. The structure of the APD is shown in schematic form in Fig 1; also shown are two APDs mounted in a $20 \mathrm{~mm}$ square plastic capsule ready for mounting on a crystal. Incident photons convert at the surface and the electrons produced generate an avalanche at the high electric field of the p-n junction. The diode structure is embedded in about 40 microns of silicon grown epitaxially on a low resistivity silicon base. The grooves etched around the edges reduce surface currents and are important for radiation hardness.

Fig 1 also indicates how the insensitivity to traversing radiation is achieved: the amplification occurs just behind the photo-conversion layer, so that only the energy deposited by particles in this thin $5 \mu \mathrm{m}$ layer is amplified, while electrons from ionising particles traversing the bulk of the silicon are not amplified. Table 1 summarises the parameters of the APDs as developed. More detailed description of the characteristics can be found in

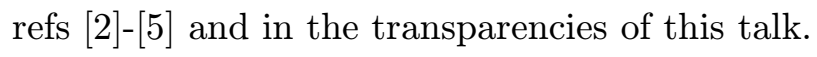

\section{Radiation Hardness and Reliability}

To maintain the performance of the CMS ECAL, the failure rate of APDs over the lifetime of the detector should be under $0.1 \%$. In order to simulate 10 years of LHC operation, the APDs are irradiated beyond the expected dose, typically with $70 \mathrm{MeV}$ protons over two hours, and then baked at $90^{\circ} \mathrm{C}$ for two weeks to simulate 10 years of aging. After these 
Active area (2 APDs per crystal)

Quantum efficiency

Light collection within 20 nsec

Operating voltage

Gain (M)

Capacitance

Serial resistance

Dark Current

Voltage sensitivity $\left(1 / \mathrm{M}^{*} \mathrm{dM} / \mathrm{dV}\right)$

Temperature sensitivity $\left(1 / \mathrm{T}^{*} \mathrm{dM} / \mathrm{dT}\right)$

Excess noise factor

Thickness sensitive to ionising particles

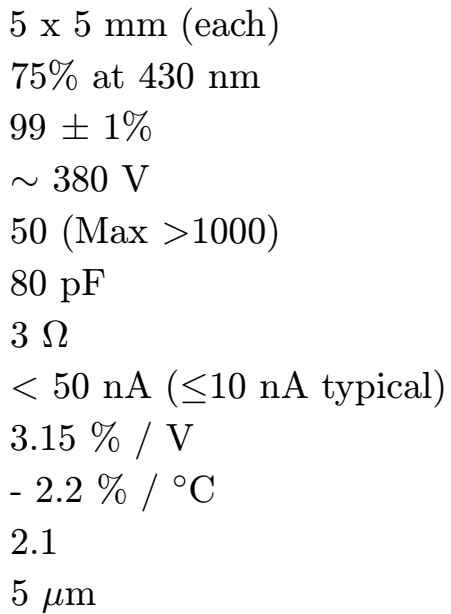

Table 1: Characteristics of the APDs for CMS

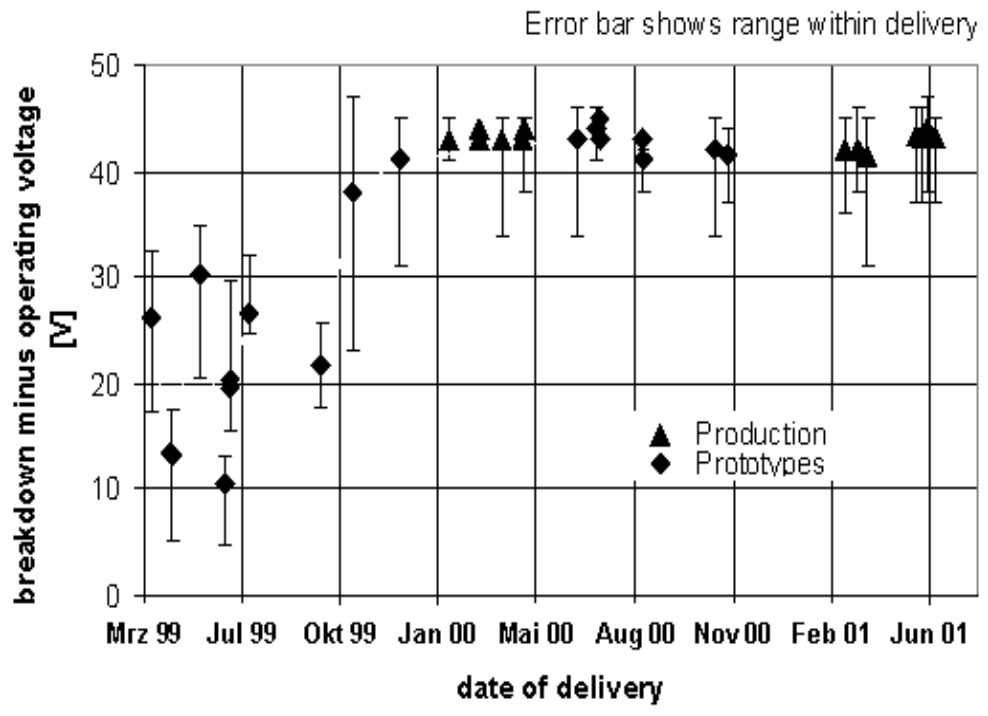

Figure 2: Mean and spread in distance between breakdown and operating voltage for APD deliveries from March 1999 until July 2001

tests, the only characteristic which changes measurably is the dark current, induced by the radiation. However, this self-anneals at room temperature, and it is estimated that after 10 years it will be only a few $\mu \mathrm{A}$.

Radiation hardness proved to be very hard to assure, and appears to be affected by a number of details. This may be due to the high internal and surface fields. One important indicator of radiation hardness was found to be the distance between breakdown voltage and operating voltage. This is now over $40 \mathrm{~V}$ (see Fig 2) for the bulk of the production (which also means that the APDs can be operated at gains of over 1000, where they still perform stably).

Fig 2 also indicates a break in production in 2000 due to reliability problems, both in the aging tests at $90^{\circ} \mathrm{C}$ and under irradiation. These proved to be two separate problems. 
$\mathrm{Vb}$ after Co irradiation minus $\mathrm{Vb}$ before

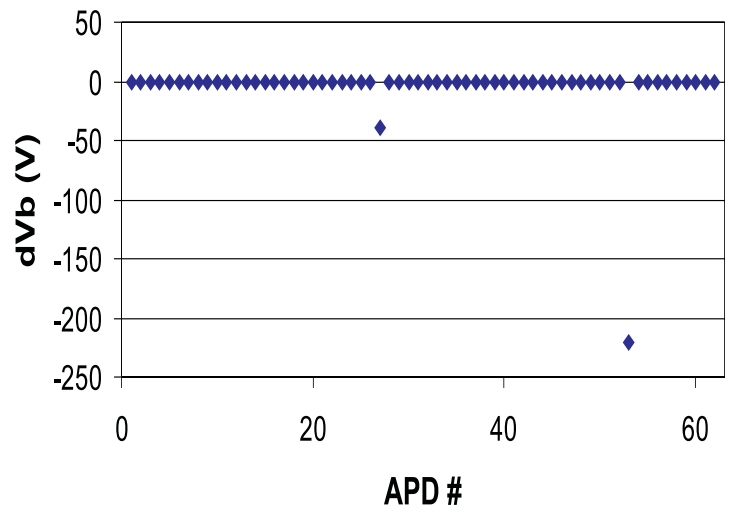

Figure 3: Change in breakdown voltage $(\mathrm{Vb})$ after cobalt irradiation

The origin of the first was soon traced by Hamamatsu and was solved. However, improving the radiation hardness required a further period of intensive R\&D by Hamamatsu and investigations by the ECAL groups. As a result, a number of detailed changes were made but not in the basic diode structure. Most of the changes were in the geometry to reduce the lateral electric fields, such as rounder corners, changed spacings and field clamps. The radiation testing procedures at PSI were also reviewed and it was established that the destructive irradiation of samples in a proton beam could be replaced by non-destructive irradiation with a $60 \mathrm{Co}$ source; this also confirmed the suspicion that the weakness is a surface problem, not in the bulk.

The conclusion of these investigations and modifications is that although the basic APD structure is radiation hard, it is very difficult to control the production processes to guarantee no problems from surface defects. Therefore it was decided to resume production (in March 2001) but with each APD screened by 5 kGray of 60Co irradiation; this is followed by 2 weeks of annealing and accelerated ageing at $90^{\circ} \mathrm{C}$, which removes nearly all the dark current induced by the radiation. Fig 3 shows the change in breakdown voltage of 62 APDs one day after irradiation, of which two APDs show big changes. Fig 4 shows the reduction in the dark current induced by the irradiation after ten days (only) at $90^{\circ} \mathrm{C}$.

APDs with changed breakdown voltage, high induced dark current or noise, either after irradiation or after two weeks in the oven, are rejected. Sample destructive testing with neutron irradiation is also carried out, which some 500 APDs have undergone without any failure. After examination of the few per cent of diodes which fail the screening, Hamamatsu believes it knows the main causes, and it appears that with screening the necessary reliability level of well over $99.9 \%$ will be achieved.

\section{Conclusion}

The APD developed by Hamamatsu meets all the requirements of the CMS ECAL. The 


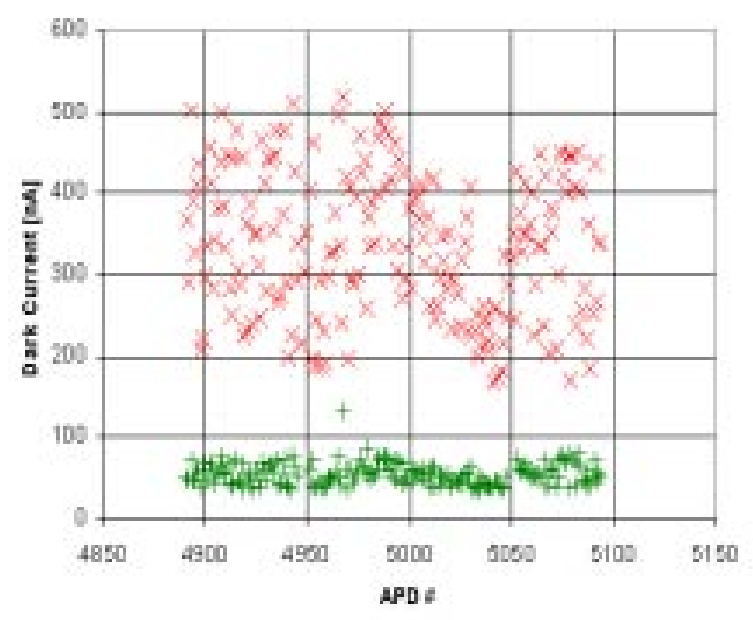

Figure 4: induced dark current immediately after the irradiation $(\mathrm{X})$ and after subsequent 10 days at $90^{\circ} \mathrm{C}(+)$

reliability problems observed after the initial deliveries have been solved, including the radiation hardness, and the mass production has resumed and is rising to the full rate required to keep to the CMS ECAL schedule.

\section{References}

[1] The CMS Electromagnetic Calorimeter Project Technical Design Report, CERN/LHCC 97-33 (1997)

[2] K. Deiters et al., Nucl. Instr. and Meth. A442 (2000) 193

[3] K. Deiters et al., Nucl. Instr. and Meth. A453 (2000) 223

[4] K. Deiters et al., Nucl. Instr. and Meth. A461 (2001) 574

[5] K. Deiters et al. Published in Cracow 2000, Electronics for LHC experiments, 221 